\title{
IMPULSE RADIATING ANTENNAS, PART II
}

\author{
Everett G. Farr, ${ }^{1}$ Carl E. Baum, ${ }^{2}$ and C. Jerald Buchenauer ${ }^{2}$ \\ ${ }^{1}$ Farr Research \\ Albuquerque, NM 87123 \\ ${ }^{2}$ Phillips Laboratory \\ Kirtland AFB, NM 87117
}

\begin{abstract}
In this continuation of our paper in the last conference proceedings ${ }^{1}$, we consider further developments in the area of Impulse Radiating Antennas (IRAs). First, we consider definitions of gain in the time domain, which are important for optimizing the performance of IRAs. A reasonable definition of gain must be equally valid in transmission as in reception. Such a definition leads naturally to a transient radar equation, which we discuss. Next, we consider how to optimize the feed impedance in a reflector IRA. If we use our simple model of IRA performance, the gain of an IRA is always better at lower impedances. But this implies larger feeds with more aperture blockage. To resolve this, we refine our simple model to account for feed blockage. We also consider the radiation pattern of IRAs, and we provide simple calculations. Finally, we provide a sample experiment which confirms our theory of IRA operation.
\end{abstract}

\section{REVIEW OF IRA DESIGN}

By now there exists a considerable body of literature concerning the design of IRA's ${ }^{1-17}$. There are two fundamental types of IRA, the reflector IRA and the lens IRA (Figure 1.1). The reflector IRA consists of a paraboloidal reflector fed by a conical TEM feed and terminated in an impedance that maintains a cardioid pattern at low frequencies. The lens IRA consists of a simple TEM horn with a lens in the aperture for focusing 25,26 . Either design is fed by a voltage source that is ideally shaped like a step function, but is in practice shaped like a fast-risetime impulse with a slower decay. In addition, either design normally has a dielectric lens at the apex to maintain voltage standoff ${ }^{26,27}$. Although there is some feed blockage associated with the reflector design, there is a considerable penalty in weight associated with the lens design. Thus, until lightweight dielectrics (real or artificial) with appropriate loss and dispersion properties are found, lens IRAs will likely be confined to applications with small apertures.

The step response of a reflector IRA on boresight consists, to first order, of a prepulse followed by an impulse. The magnitude of the prepulse is determined by transmission line techniques ${ }^{1,8}$, and it lasts for the round-trip transit time of the feed, $2 F / c$, where $F$ is the focal length of the reflector, and $c$ is the speed of light. The magnitude of the impulse is found by aperture integration ${ }^{1,5}$. The total response is

$$
E(r, t)=\frac{V_{o}}{r} \frac{D}{4 \pi c f_{g}}\left\{\delta_{a}(t-2 F / c)-\frac{c}{2 F}[u(t)-u(t-2 F / c)]\right\}
$$

where $D$ is the diameter of the reflector, $f_{g}=Z_{\text {feed }} / Z_{o}, Z_{O}$ is the impedance of free space, $V_{o}$ is the magnitude of the driving voltage step launched onto the feed, $r$ is the distance away from the antenna on boresight, and $u(t)$ is the Heaviside step function. Furthermore, $\delta_{a}(t)$ is the approximate Dirac delta function $^{3}$, which approaches a true Dirac delta function as $r$ approaches infinity. This is a high-impedance approximation based on the aperture integration described by Baum ${ }^{5}$. Later, we provide a correction for lower impedances. Note that the above equation can be expressed in terms of an arbitrary driving voltage as 


$$
E(r, t)=\frac{D}{4 \pi r c f_{g}}\left\{\frac{d V(t-2 F / c)}{d t}-\frac{c}{2 F}[V(t)-V(t-2 F / c)]\right\}
$$

where $V(t)$ is the voltage launched onto the feed.
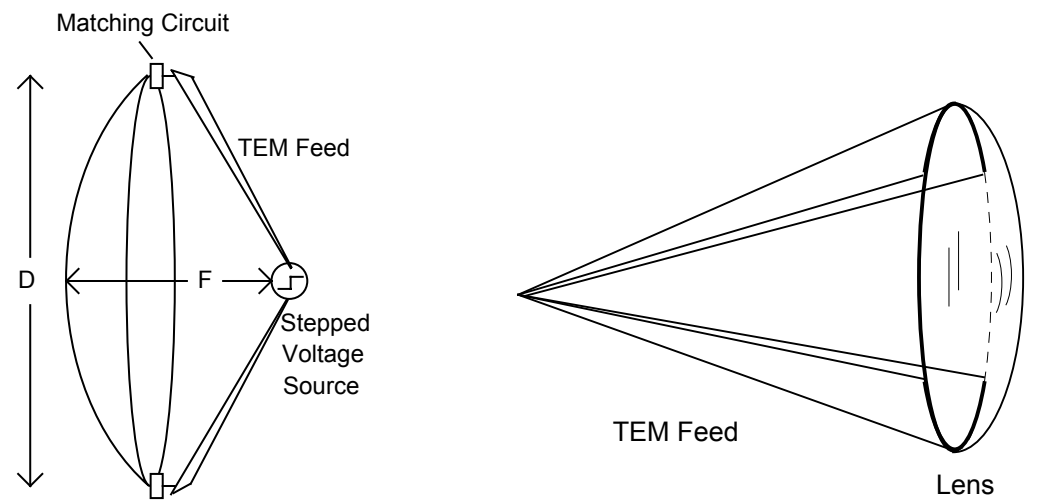

Figure 1.1. A reflector IRA (left) and a lens IRA (right).

\section{GAIN DEFINITION IN THE TIME DOMAIN}

If we are to optimize the feed impedance of the IRA, it will be necessary to provide a good definition of that quantity which is to be optimized. The definition of gain in the frequency domain is already well established as an IEEE standard ${ }^{18}$. However, no analogous definition has been developed in the time domain. There have been a number of attempts to clarify this point, however, none have provided a definition that is consistent with reciprocity. That is, none of the definitions are as meaningful in reception as in transmission. We propose here a definition that meets this criterion.

Since the exact definition of gain is so critical, it is useful to consider its definition in the frequency domain. According to the IEEE standard the definition is as follows:

gain; absolute gain (of an antenna in a given direction). The ratio of the radiation intensity, in a given direction, to the radiation intensity that would be obtained if the power accepted by the antenna were radiated isotropically.

NOTES: (1) Gain does not include losses arising from impedance and polarization mismatches. (2) The radiation intensity corresponding to the isotropically radiated power is equal to the power accepted by the antenna divided by $4 \pi$. (3) If an antenna is without dissipative loss, then in any given direction, its gain is equal to its directivity. (4) If the direction is not specified, the direction of maximum radiation intensity is implied. (5) The term absolute gain is used in those instances where added emphasis is required to distinguish gain from relative gain; for example, absolute gain measurements.

directivity, partial (of an antenna for a given polarization). In a given direction, that part of the radiation intensity corresponding to a given polarization divided by the total radiation intensity averaged over all directions.

NOTE: The (total) directivity of an antenna, in a specified direction, is the sum of the partial directivities for any two orthogonal polarizations.

radiation pattern; antenna pattern. The spatial distribution of a quantity which characterizes the electromagnetic field generated by an antenna.

NOTES: (1) The distribution can be expressed as a mathematical function or as a graphical representation. (2) The quantities which are most often used to characterize the radiation from an antenna are proportional to, or equal to, power flux density, radiation intensity, directivity, phase, polarization, and field strength. (3) The spatial distribution over any surface or path is also an antenna pattern. (4) When the amplitude or relative amplitude of a specified component of the electric field vector is plotted graphically, it is called an amplitude pattern, field pattern, or voltage pattern. When the square of the amplitude or relative amplitude is plotted, it is called a power pattern. (5) When the quantity is not specified, an amplitude or power pattern is assumed.

Let us point out some features of these definitions. First, we note that gain is independent of source mismatch. In fact, antenna gain is independent of all source parameters with the exception of frequency. In the time domain, we might consider replacing a dependence upon frequency with a dependence upon risetime, peak derivative, or Full Width Half Max (FWHM) of the driving function. Second, we note that the 
definitions of gain and directivity assume one is looking at the total radiation in a given direction. If one were considering the effects of polarization, one would use a partial gain, or partial directivity. Next, we note that gain is normalized to the "power accepted by the antenna," while directivity is normalized to the "total power radiated by the antenna." Antenna gain takes into account antenna losses, while directivity does not.

It is interesting to note here that all the terms here are defined solely for transmit mode--there is no mention of the antenna being used as a receiver. This is all that is needed because there is a simple relationship between an antenna's transmitting and receiving properties in the frequency domain. To convert a transmit pattern to a receive pattern, one merely multiplies by $1 / j \omega$, which is a constant in the frequency domain where $s=j \omega$. However, this corresponds to an integral in the time domain, so in the time domain the conversion is not as simple.

Let us associate some equations with the above definitions. According to Stutzman and Thiele ${ }^{19}$ gain is

$$
G(\theta, \phi, \omega)=\frac{4 \pi U(\theta, \phi, \omega)}{P_{\text {in }}(\omega)}
$$

where $U(\theta, \phi, \omega)$ is the radiation intensity in Watts/steradian, and $P_{\text {in }}(\omega)$ is the power accepted by the antenna. Furthermore, antenna pattern is

$$
F(\theta, \phi, \omega)=\frac{U(\theta, \phi, \omega)}{U\left(\theta_{\max }, \phi_{\max }, \omega\right)}
$$

where $U\left(\epsilon_{\max }, \phi_{\max }, \omega\right)$ is the radiation intensity in the direction of maximum radiation (boresight).

In order to extend the definition of gain into the time domain, we must express the radiated and received fields in terms of the incident voltage (in transmission) and the incident field (in reception). The diagrams showing the relevant quantities are shown in Figure 2.1. Note that there is a resistive load that is matched to the characteristic impedance of any feed transmission line attached to the antenna port. (This will also be matched to the IRA feed, which is itself a conical transmission line.) This is analogous to the use of scattering parameters in circuit theory.

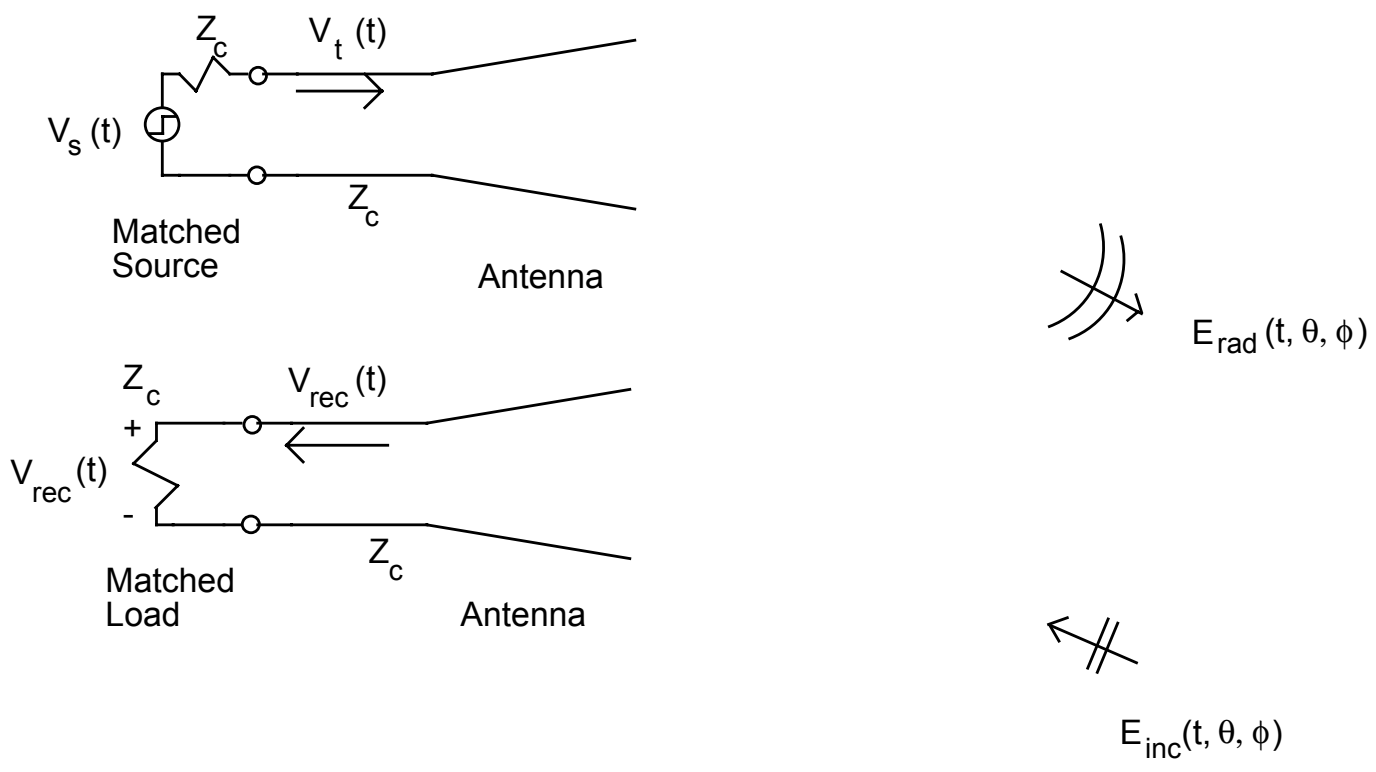

Figure 2.1. A transient antenna in transmit mode (top) and receive mode (bottom).

First we describe the relevant equations in the frequency domain. Because of the resistive termination matched to the feed line, in transmission $V_{t}(t)=V_{s}(t) / 2$. Instead of referring to port voltages, we will refer to voltage waves, in the spirit of $S$-parameters in microwave theory. Thus, the transmitted and radiated fields are, according to $\mathrm{Baum}^{7}$

Transmit

$\widetilde{\vec{E}}_{\text {rad }}(r, s)=\frac{e^{-\gamma r}}{r} \widetilde{\vec{F}}_{t}\left(1_{r}, s\right) \widetilde{V}_{t}(s)$

Receive

$$
\widetilde{V}_{\text {rec }}(s)=\tilde{\vec{h}}_{t}\left(\overrightarrow{1}_{i}, s\right) \cdot \tilde{\vec{E}}_{\text {inc }}(s)
$$


Reciprocity

$$
\begin{aligned}
& \tilde{\vec{F}}_{t}\left(\overrightarrow{1}_{r}, s\right)=\frac{s \mu_{o}}{2 \pi Z_{c}} \stackrel{\leftrightarrow}{1}_{r} \cdot \tilde{\vec{h}}_{t}\left(-\overrightarrow{1}_{r}, s\right) \\
& \stackrel{\leftrightarrow}{1}_{r}=\stackrel{\leftrightarrow}{1}-\overrightarrow{1}_{r} \overrightarrow{1}_{r}, \quad \stackrel{\leftrightarrow}{1}=\overrightarrow{1}_{x} \overrightarrow{1}_{x}+\overrightarrow{1}_{y} \overrightarrow{1}_{y}+\overrightarrow{1}_{y} \overrightarrow{1}_{y},
\end{aligned}
$$

where $\overrightarrow{1}_{r}$ is the direction of radiation and $\gamma=s / c$.. The time domain analogs of these equations are

Transmit

$$
\begin{aligned}
& \vec{E}_{\text {rad }}(\vec{r}, t)=\left[\frac{1}{r} \vec{F}_{t}\left(\overrightarrow{1}_{r}, t\right)\right] V_{t}(t-r / c) \\
& r \vec{E}_{\text {rad }}(\vec{r}, t)=\left[\int_{0}^{t} \vec{F}_{t}\left(\overrightarrow{1}_{r}, t^{\prime}\right) d t^{\prime}\right] \frac{d V_{t}(t-r / c)}{d t}
\end{aligned}
$$

Receive

$$
V_{\text {rec }}(t)=\vec{h}_{t}\left(\overrightarrow{1}_{i}, t\right) \cdot \vec{E}_{\text {inc }}(t)
$$

Reciprocity

$$
\vec{F}_{t}\left(\overrightarrow{1}_{r}, t\right)=\frac{\mu_{o}}{2 \pi Z_{c}} \overleftrightarrow{1}_{r} \cdot \frac{d}{d t} \vec{h}_{t}\left(-\overrightarrow{1}_{r}, t\right)
$$

where the ${ }^{\circ}$ operator indicates a convolution and the dot product convolution operator $\sqcup$ implies a sum of the convolution of each component of the vectors. Note that the units of $\vec{h}(t)$ are meters/second. Note also that the function $\int_{0}^{t} \vec{F}_{t}\left(\overrightarrow{1}_{r}, t^{\prime}\right) d t^{\prime}$ is the step response in transmission, which has been characterized for reflector IRA's (earlier in this paper) and for TEM horns by Farr and Baum ${ }^{9}$. Finally, note that $\vec{h}_{t}\left(\overrightarrow{1}_{i}, t\right)$ is just the step response in transmission times some constants.

We can now drive the antenna with a standard waveshape, such as the integral of a Gaussian (in transmission) or a Gaussian (in reception). Because of the above reciprocity relationship in the time domain we can establish a correlation between the transmit and receive cases. We now propose a gain defined in terms of norms as

$$
G(\theta, \phi)=\frac{1}{\sqrt{f_{g}}} \frac{\left\|V_{r e c}(t)\right\|}{\left\|\vec{E}_{\text {inc }}(\theta, \phi, t) \cdot \overrightarrow{1}_{e}\right\|} \quad G(\theta, \phi)=\lim _{r \rightarrow \infty} \frac{2 \pi c \sqrt{f_{g}\left\|r \vec{E}_{\text {rad }}(\theta, \phi, t) \cdot \overrightarrow{1}_{e}\right\|}}{\left\|d V_{\text {inc }}(t) / d t\right\|}
$$

\section{Transmission}

where $\overrightarrow{1}_{e}$ is one of two orthogonal polarizations. The above two definitions are guaranteed to be equal if the driving voltage waveshape in transmission is the integral of the incident electric field in reception. One can think of the norm of a function as one of several commonly used characteristics of a time domain function, such as the peak of the function ( $\infty$-norm), the integral of magnitude of the function (1-norm), or the square root of " energy" in the function (2-norm). By way of review, a norm must satisfy three fundamental properties,

$$
\|f(t)\|\left\{\begin{array}{cc}
=0 & \text { iff } f(t) \equiv 0 \\
>0 & \text { otherwise }
\end{array}, \quad\|\alpha f(t)\|=|\alpha|\|f(t)\|, \quad\|f(t)+g(t)\| \leq\|f(t)\|+\|g(t)\|\right.
$$

Recall also the definition of $p$-norms,

$$
\|f(t)\|_{p} \equiv\left(\int_{-\infty}^{\infty}|f(t)|^{p} d t\right)^{1 / p}, \quad\|f(t)\|_{\infty} \equiv \sup _{t}|f(t)|
$$

The choice of the norm will usually be tied to the experimental system. Thus, if a transient radar receiver responds to the peak magnitude of the received signal, then one should use the $\infty$-norm in the gain definitions.

There are a number of other characteristics of this gain definition that should be noted. The units of gain are meters, which is different than the unitless gain of the frequency domain. Furthermore, the transient gain is dependent upon (1) the shape of the waveform, and (2) the choice of the norm. Thus, a transient gain must always be specified in relation to these two parameters. Note also that gain is a function of risetime (of step-like waveform in transmission) or FWHM (of impulse-like waveform in reception). Thus, gain is a function of risetime in the time domain, as it is a function of frequency in the frequency domain for $s=j \omega$. Note also that it is trivial to extend these concepts to two polarizations. Furthermore, one can assign the term antenna pattern to the variation of this gain as a function of angle.

One can apply bounds to all of the convolutions shown in (2.4), by taking the norm of both sides of the equation. For example, consider the equation for reception in (2.4). If we take the norm of both sides of the equation we find an upper bound on the received voltage as 


$$
\left\|V_{\text {rec }}(t)\right\|=\left\|\left[\vec{h}_{t}\left(\overrightarrow{1}_{i}, t\right) \cdot\right] \vec{E}_{\text {inc }}(t)\right\| \leq\left\|\vec{h}_{t}\left(\overrightarrow{1}_{i}, t\right) \cdot\right\| \vec{E}_{\text {inc }}(t) \|
$$

where $\left\|\vec{h}_{t}\left(\overrightarrow{1}_{i}, t\right) \cdot\right\|$ has to be interpreted in the sense of a norm of a dyadic (matrix) convolution. This is much simpler to understand one polarization at a time, so if we assume $\vec{E}_{\text {inc }}(t)=\overrightarrow{1}_{p} E_{\text {inc }}(t)$ then

$$
\left\|V_{\text {rec }}(t)\right\|=\left\|\left[\vec{h}_{t}\left(\overrightarrow{1}_{i}, t\right) \cdot \overrightarrow{1_{p}}\right] E_{\text {inc }}(t)\right\| \leq\left\|\vec{h}_{t}\left(\overrightarrow{1}_{i}, t\right) \cdot \overrightarrow{1}_{p}\right\|\left\|E_{\text {inc }}(t)\right\|
$$

Thus, if some norm is applied to the incident field and to the antenna system response, then one can place an upper bound on the norm of the received voltage. Finally, we invoke the property that the $p$-norm of a convolution operator is less than or equal to the 1-norm of the impulse response ${ }^{24}$, or $\|g(t)\| \leq\|g(t)\|_{1}$. Thus we find, for a single polarization,

$$
\left\|V_{\text {rec }}(t)\right\|_{p} \leq\left\|\overrightarrow{h_{t}}\left(\overrightarrow{1}_{i}, t\right) \cdot \overrightarrow{1}_{p}\right\|_{1}\left\|E_{\text {inc }}(t)\right\|_{p}
$$

This establishes a bound on the $p$-norm of the received voltage for a given incident field polarization. Note that in general we will not want to restrict ourselves to using $p$-norms, but if one chooses to one can invoke a nice simplification. Similar bounds can be applied to the transmit equation.

It may be useful to compare our gain definitions to those proposed by other authors. O. Allen, et al, have proposed a definition of gain in transmission mode as ${ }^{20}$

$$
G(\theta, \phi)=\frac{4 \pi r^{2}}{Z_{o}} \frac{\int_{-\infty}^{\infty}\left|E_{\text {trans }}(\theta, \phi)\right|^{2} d t}{\int_{-\infty}^{\infty} V_{\text {input }}(t) I_{\text {input }}(t) d t}
$$

This is somewhat related to our earlier definition of gain if one uses the square of the 2-norm, however, there is no time derivative in the denominator. It is simple to show that without a time derivative, this definition of gain is not meaningful in receive mode.

A related family of figures of merit has been proposed by R. Ziolkowski, for use in near field arrays $^{21}$. He proposes a figure of merit that relates the radiated energy at a location in the near field to the total energy accepted by the array. This is similar to the definition of gain proposed by Allen et al., although somewhat different in detail. Ziolkowski does not consider reciprocity, but it is not yet clear how to do so in the near field. Recall that even in the frequency domain, gain is not defined in the near field. Thus, these figures of merit were likely not meant to be a rigorous replacement for antenna gain in the time domain. It should also be noted that Ziolkowski uses norm concepts to arrive at his figure of merit, much in the same spirit as we use them here.

Now that we have established a gain definition, it is appropriate to apply it to a transient radar equation. Consider Figure 2.2, which shows a transmitting antenna, a scattering object, and a receiving antenna. For the moment we provide the equations for the most general case, including all polarizations and allowing for different transmit and receive antennas. Later, we will simplify by allowing only a single polarization, with identical transmit and receive antennas in the same location.

One can calculate the radiated field in terms of the voltage wave launched onto the antenna feed as

$$
\vec{E}^{r a d}\left(r, \overrightarrow{1}_{r}, t\right)=\frac{\mu_{O}}{2 \pi r Z_{c}} \overleftrightarrow{1}_{r} \cdot \vec{h}_{\text {trans }}\left(\overrightarrow{1}_{r}, t\right) \frac{d V^{i n c}(t-r / c)}{d t}
$$

where $r$ is the distance from the transmitting antenna to the scatterer, $Z_{c}$ is the feed impedance, and $\overrightarrow{1_{r}}$ is the direction of the radiated field. The scattered field is

$$
\vec{E}^{s c a t}\left(r^{\prime}, \overrightarrow{1}_{i}, t\right)=\frac{1}{4 \pi r} \stackrel{\leftrightarrow}{\Lambda}\left(\overrightarrow{1}_{r}, \overrightarrow{1}_{i}, t\right) \cdot \vec{E}^{r a d}\left(r, \overrightarrow{1}_{r}, t^{\prime}-\left(r+r^{\prime}\right) / c\right)
$$




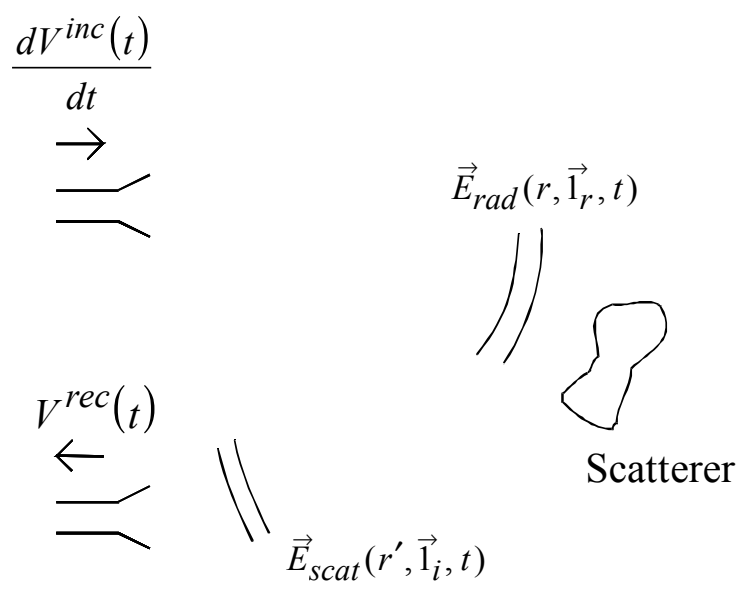

Figure 2.2. The configuration for a transient radar equation.

where $r^{\prime}$ is the distance from the scatterer to the receiving antenna, $\overrightarrow{1}_{i}$ is the angle of incidence of the scattered wave on the receiver, and $\overleftrightarrow{\Lambda}\left(\overrightarrow{1}_{r}, \overrightarrow{1}_{i}, t\right)$ is the scattering dyadic length as a function of time ${ }^{23}$. Finally, the received voltage wave is

$$
V^{r e c}(t)=\vec{h}_{r e c}\left(\overrightarrow{1}_{i}, t\right) \cdot \vec{E}^{s c a t}\left(r^{\prime}, \overrightarrow{1}_{i}, t-\left(r+r^{\prime}\right) / c\right)
$$

Putting it all together, we find a total response of

$$
V^{r e c}(t)=\frac{1}{8 \pi^{2} r^{2} c f_{g}} \vec{h}_{r e c}\left(\overrightarrow{1}_{i}, t\right) \cdot \stackrel{\leftrightarrow}{\Lambda}\left(\overrightarrow{1}_{r}, \overrightarrow{1}_{i}, t\right) \cdot \vec{h}_{\text {trans }}\left(\overrightarrow{1}_{r}, t\right) \frac{d V^{i n c}\left(t-\left(r+r^{\prime}\right) / c\right)}{d t}
$$

Finally, the above equation may be bounded by

$$
\left\|V^{r e c}(t)\right\| \leq \frac{1}{8 \pi^{2} r^{2} c f_{g}}\left\|\vec{h}_{r e c}\left(\overrightarrow{1}_{r}, t\right)\right\|\left\|\overleftrightarrow{\Lambda}\left(\overrightarrow{1}_{r}, \overrightarrow{1}_{i}, t\right)\right\|\left\|\vec{h}_{\text {trans }}\left(\overrightarrow{1}_{i}, t\right)\right\|\left\|\frac{d V^{i n c}(t)}{d t}\right\|
$$

These last two equations may be considered a time domain analog of the standard radar equation in the frequency domain.

Let us now simplify the above equations in a variety of ways. First, we assume that the transmit and receive antennas are located in the same position (monostatic case), and that their characteristics are identical. Thus, $\overrightarrow{1}_{r}=-\overrightarrow{1}_{i}$ and $\vec{h}_{r e c}\left(\overrightarrow{1}_{r}, t\right)=\vec{h}_{\text {trans }}\left(\overrightarrow{1}_{r}, t\right)$. In addition, we consider for simplicity only one component of the radiated and received field, for example, the horizontal or $h$ component, and assume that the vertical or $v$ component is zero We can then convert the radar equation to

$$
V^{r e c}(t)=\frac{1}{8 \pi^{2} r^{2} c f_{g}} h_{h}(t) \quad \Lambda_{h h}(t) \quad h_{h}(t) \quad \frac{d V^{i n c}\left(t-\left(r+r^{\prime}\right) / c\right)}{d t}
$$

where the convolutions commute, if one wishes. One can then establish a bound on the received signal,

$$
\left\|V^{r e c}(t)\right\| \leq \frac{1}{8 \pi^{2} r^{2} c f_{g}}\left\|h_{h}(t) h_{h}(t)\right\|\left\|\Lambda_{h h}(t)\right\|\left\|\frac{d V^{i n c}(t)}{d t}\right\|
$$

Note the interesting double convolution $h_{h}(t) h_{h}(t)$, whose norm is a part of the bound on the received voltage.

\section{OPTIMIZATION OF REFLECTOR IRA FEED IMPEDANCE}

If one applies our definition of gain to the impulsive portion of the simple model of the reflector IRA, one arrives at a gain of $D /\left(2 f_{g}^{1 / 2}\right)$. This suggests that the gain always gets better at lower feed impedances. This leads to increasingly fatter antenna feeds with more feed blockage, so we reach a contradiction.

The problem arises because the approximate model for the impulsive portion of the field was developed by ignoring feed blockage ${ }^{5}$. Recall that the surface integral was calculated by converting an aperture surface integral to a contour integral around the border of the aperture. Consider Figure 3.1, which 
shows one-fourth of the aperture that must be integrated. On the left is the contour that was integrated originally ${ }^{5}$. A better aperture (for two arms), which accounts for feed blockage is shown in the center. Often one will use four arms instead of two, in order to reduce the feed impedance, so we also show on the right a contour appropriate for the four-arm configuration.
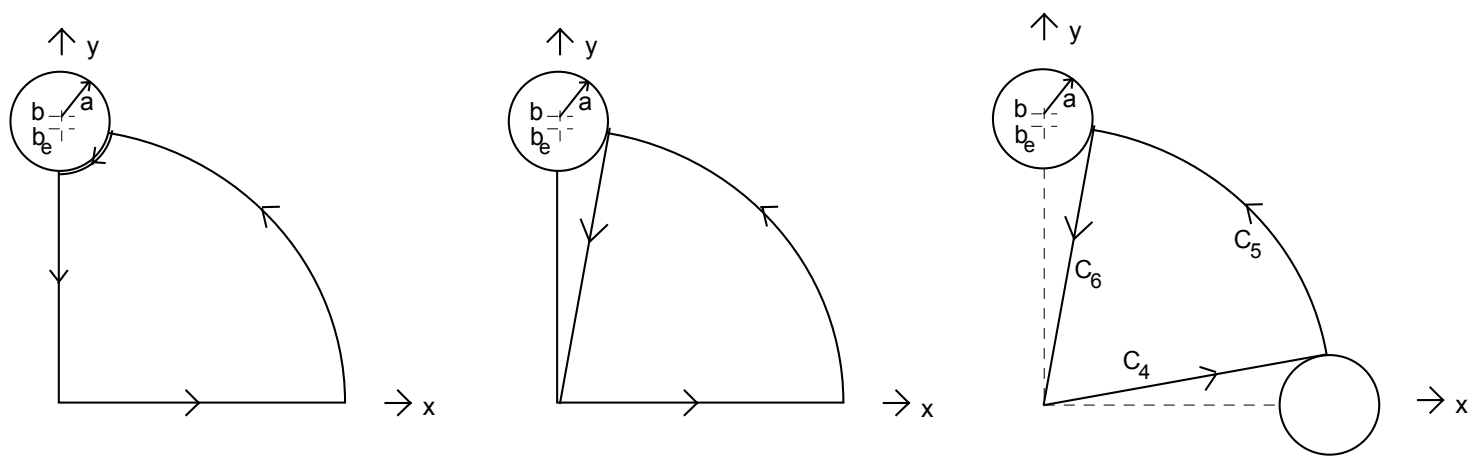

Figure 3.1. The old contour for the aperture integration (left), and the corrected contours for a two-arm (center) and four-arm (right) configurations.

To clarify the point, we review some of the theory of radiation from apertures. Consider the an aperture field that turns on suddenly with a step-function time dependence. We construct a complex aperture electric field function whose real and imaginary parts correspond to the $x$ and $y$ components of the aperture electric field. This complex field is expressed in terms of a complex potential function ${ }^{5}$. Thus,

$$
\begin{aligned}
& E(x, y)=E(\zeta)=E_{x}-j E_{y}=-\frac{V_{O}}{\Delta u} \frac{d w(\zeta)}{d \zeta} \\
& \zeta=x+j y, \quad w(\zeta)=u(\zeta)+j v(\zeta), \quad f_{g}=\Delta u / \Delta v
\end{aligned}
$$

where the complex potential function can be found in Smythe ${ }^{22}$. In the above formulation, $\Delta v$ is the change in $v$ around one of the conductors, and $\Delta u$ is the difference in $u$ from one conductor to the other. It was also shown that the radiated field on boresight is ${ }^{5}$

$$
\begin{aligned}
& E^{r a d}(r, t)=\frac{V_{o}}{r} \frac{h_{a}}{2 \pi c f_{g}} \delta_{a}(t-r / c) \\
& h_{a}=-\frac{f_{g}}{V_{o}} \iint_{S_{a}} E_{y}(x, y) d x d y=-\frac{1}{\Delta v} \oint_{C_{a}} v(y) d y
\end{aligned}
$$

In the above equation, $S_{a}$ is the portion of the aperture that is not blocked by the feed, and $C_{a}$ is the contour around this aperture. All contour integrals in this paper are in the counterclockwise direction. A highimpedance approximation was made which claimed that feed blockage could be ignored, and that the portion of the contour integral along the conducting wire was very small. Under this approximation the integral is calculated as $h_{a}=D / 2$. This provided the impulsive portion of the radiated field in (1.1) A more accurate integral, however, excludes the portion of the aperture integral that is blocked by the feed, as shown in the center and right of Figure 3.1. Note that Figure 3.1 shows one-fourth of $C_{a}$.

We have plotted the value of $h_{a}$ for a two-arm circular cone configuration using the improved contour in Figure 3.2. Our plot is for a radius of 1 meter. The gain, using the 1 -norm, is just $h_{a} / f_{g}^{1 / 2}$ and this is also plotted in Figure 3.2, again for a reflector with a radius of 1 meter. The gain is a slowly varying function that peaks around $312 \Omega$ at a value of $0.85 \mathrm{~m}$. Note that for this type of antenna, it is not necessary to specify the driving waveform in order to specify a gain, since all waveforms give the same result.

If one uses four arms, then there is slightly more feed blockage, but the input impedance is reduced by a factor of two. This makes it considerably easier to build a balun to match the impedance of the feed line, which is typically $50 \Omega$. We plot for this configuration $h_{a}$ and gain as a function of feed impedance in Figure 3.3. Note that the gain peaks at $406 \Omega$, at a value of $0.81 \mathrm{~m}$. If one has two $400 \Omega$ feeds in parallel, the net input impedance is $200 \Omega$, which is a convenient value of impedance for the output of a balun with a 1:4 impedance ratio ${ }^{4}$. Thus, the peak in gain near $400 \Omega$ is fortuitous.

This concept can be extended to a variety of other cases, including different feed geometries, for example, feeds constructed out of flat plates that are either coplanar or facing. Many of these other cases have been developed by Farr ${ }^{13}$. 

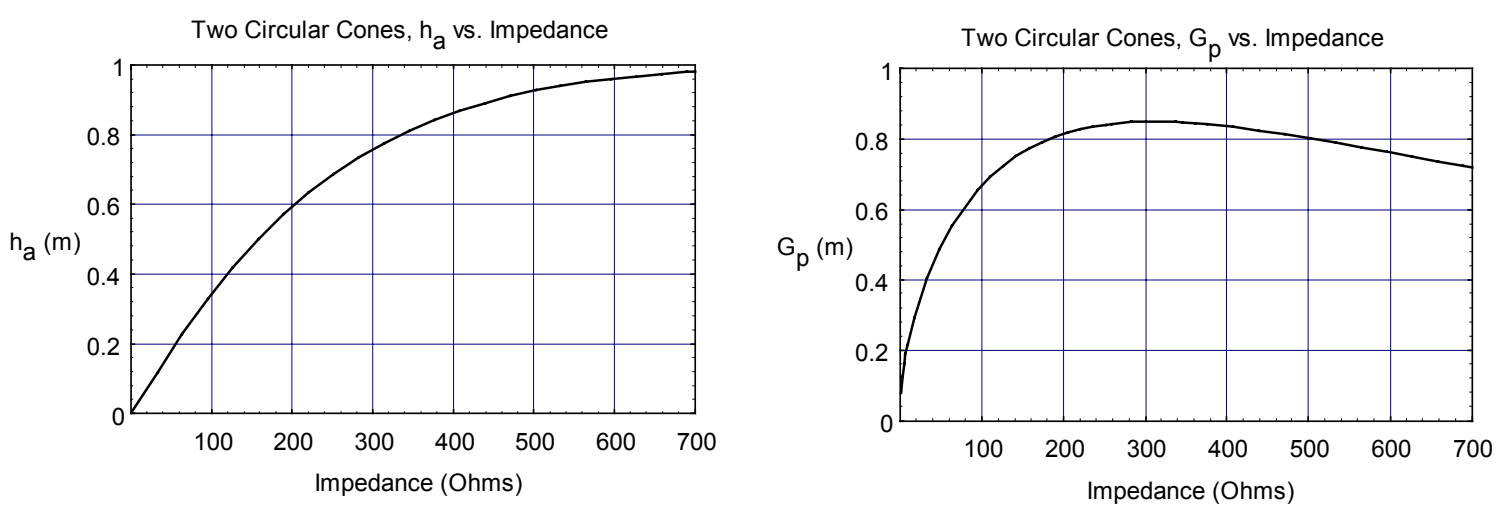

Figure 3.2. Plots of the effective height $h_{a}$ of the aperture (left) and the gain (right) as a function of feed impedance for a reflector IRA with two circular cone feeds.
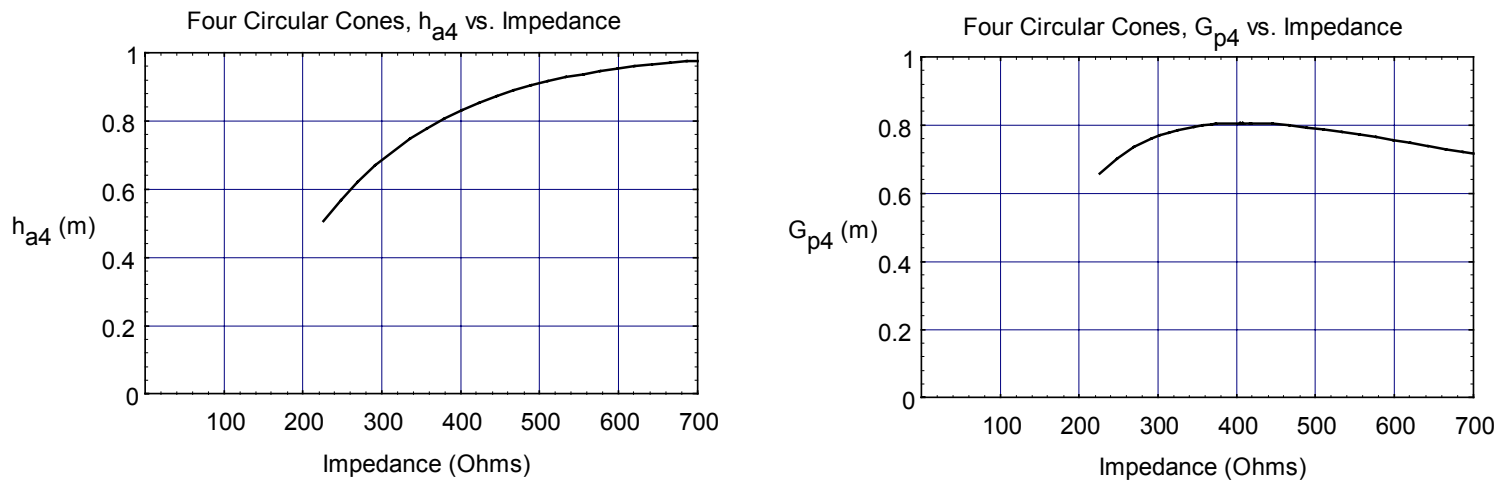

Figure 3.3. Plots of the effective height $h_{a}$ of the aperture (left) and the gain (right) as a function of feed impedance for a reflector IRA with four circular cone feeds.

\section{ANTENNA PATTERN OF A REFLECTOR IRA}

The simplest definition of an antenna pattern in the time domain is simply to plot the gain as we defined it earlier in this paper as a function of angle. In order to calculate the gain as a function of angle, one must first calculate the radiated field as a function of angle. Recall that to calculate the field on boresight, we used the integral of the electric field over the entire aperture. To calculate the step-response radiated field off boresight, one can show from time delay considerations that the radiated field off-boresight at a given point in time is proportional to a line integral of the electric field over the aperture. This radiated field varies as the value of the line integral sweeping across the aperture. (Figure 4.1). Thus, the step-response radiated far fields in the $H$ - and $E$-planes are proportional to the normalized potentials $\Phi^{(h)}$ and $\Phi^{(e)}$, which are defined as

$$
\Phi^{(h)}(x)=\frac{-1}{V_{o}} \int_{C_{1}(x)} E_{y} d y \quad, \quad \Phi^{(e)}(y)=\frac{-1}{V_{o}} \int_{C_{2}(y)} E_{y} d x
$$

where the contours $C_{1}(x)$ and $C_{2}(y)$ are shown in Figure 5.1.
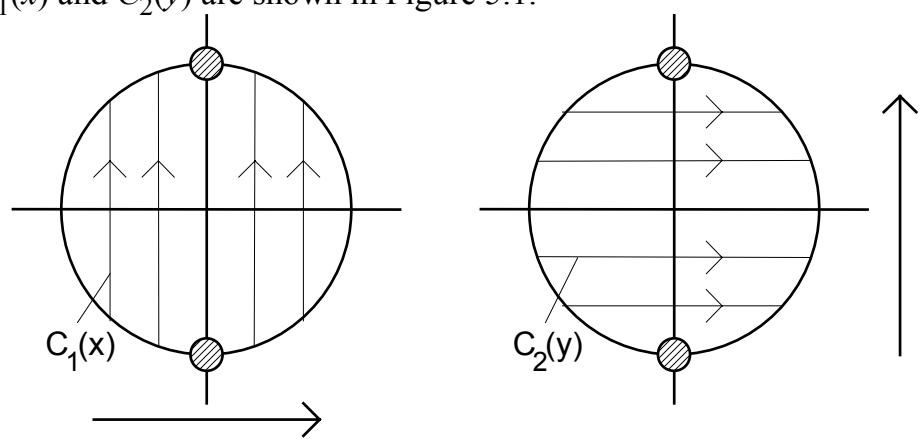

Figure 4.1. The location of the line integrals $C_{1}(x)$ and $C_{2}(y)$ as a function of position in the aperture. function is ${ }^{22}$,

To evaluate the above integrals, we use the fields and potentials for round wires. Thus the potential

$$
w(\zeta)=2 j \operatorname{arccot}(\zeta / a)=\ln \left(\frac{\zeta-j a}{\zeta+j a}\right), \quad \zeta=x+j y
$$


and the fields are calculated from this potential from (3.1). After substituting into (4.1), we find

$$
\begin{aligned}
\Phi^{(h)}(x) & = \begin{cases}1 /\left(\pi f_{g}\right) \operatorname{arcsech}(-x / a) & -a \leq x \leq-a \operatorname{sech}\left(\pi f_{g}\right) \\
1 & -a \operatorname{sech}\left(\pi f_{g}\right) \leq x \leq a \operatorname{sech}\left(\pi f_{g}\right) \\
1 /\left(\pi f_{g}\right) \operatorname{arcsech}(x / a) & a \operatorname{sech}\left(\pi f_{g}\right) \leq x \leq a\end{cases} \\
\Phi^{(e)}(y) & =\left\{\begin{array}{cl}
1 /\left(2 f_{g}\right) & |y| \leq a \\
0 & \text { else }
\end{array}\right.
\end{aligned}
$$

The details of the radiated field calculation have been worked out by Farr and Baum ${ }^{13}$. The final result for the radiated field in the $E$ - and $H$-planes as a function of time is

$$
\vec{E}^{(h)}(r, t)=\overrightarrow{1}_{y} \frac{-V_{o}}{r} \frac{\cot (\theta)}{2 \pi} \Phi^{(h)}\left(\frac{c t}{\sin (\theta)}\right), \vec{E}^{(e)}(r, \theta, t)= \pm \overrightarrow{1}_{\theta} \frac{-V_{o}}{r} \frac{1}{2 \pi \sin (\theta)} \Phi^{(e)}\left(\frac{c t}{\sin (\theta)}\right)
$$

where $\theta$ is the angle from boresight. These are plotted in Figure 4.2 for round-wire feeds at $400 \Omega$. Note that feed blockage has been ignored in this formulation.
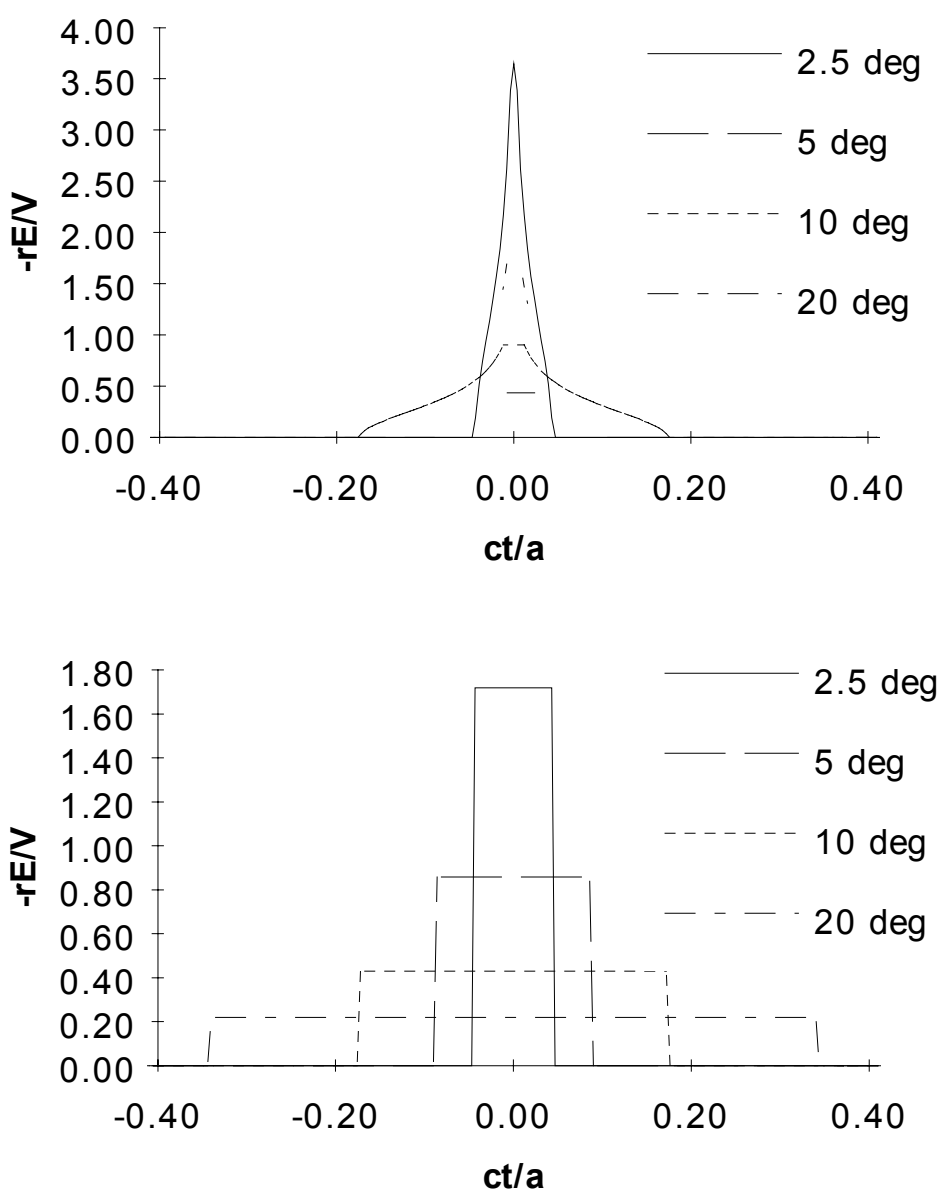

Figure 4.2. Step response of the radiated field in the H-plane (top) and E-plane (bottom).

Now that we have the step response, we must find the band-limited step response before calculating the gain. This is necessary because the step response is ill-behaved on boresight, where it becomes an approximate delta function. Since the $p$-norm of the approximate delta function only exists for the 1-norm, we have more flexibility in our choice of norms by converting to the band-limited response. We do so by driving the antenna with the integral of a Gaussian waveform with a finite risetime. We then convolve the step response with derivative of the driving voltage (a Gaussian). Thus, we have for the driving voltage

$$
\frac{d V(t)}{d t}=\frac{V_{o}}{t_{d}} e^{-\pi\left(t / t_{d}\right)^{2}}, \quad t_{F W H M}=0.940 t_{d}
$$




$$
V(t)=\int_{-\infty}^{t} \frac{d V\left(t^{\prime}\right)}{d t^{\prime}} d t^{\prime}, \quad t_{10-90}=1.023 t_{d}
$$

where $t_{F W H M}$ is the Full Width Half Max of $d V / d t$, and $t_{10-90}$ is the $10-90 \%$ risetime of $V(t)$. Note that we have expressed this conveniently in terms of the derivative risetime, which is inversely proportional to the radiated field for these types of antennas. The definition of the derivative risetime of a waveform is

$$
t_{d}=\frac{\max (V(t))}{\max (d V(t) / d t)}
$$

The radiated field is now calculated simply from

$$
E(r, \theta, \phi, t)=\frac{1}{V_{o}} \frac{d V(t)}{d t} E^{s t e p}(r, \theta, \phi, t)
$$

where $E^{\text {step }}(r, \theta, \phi, t)$ is the step response in the $E$ - or $H$-plane, as calculated above. We can reduce the number of cases that need to be calculated by defining a rise parameter $T_{d}$ as

$$
T_{d}=\frac{t_{d}}{t_{a}}=\frac{c t_{d}}{a}
$$

where $a$ is the aperture radius. All problems with equal rise parameters have the same shape radiated field. Thus, all problems with the same $T_{d}$ can be characterized by a single curve with proper scaling.

The antenna pattern is now just our previously defined gain measured as a function of angle in the $E$ and $H$-planes, as defined in the transmission case of (2.5). Note that the gain has units of meters, and that the gain is normalized to the radius of the antenna. For our calculations we have used the $\infty$-norm, or the peak of the radiated field, although many other norms might be suitable. Note also that because we have been careful with our definition of transient gain, our results also apply to the antenna in receive mode with a Gaussian incident field.

A sample problem has been solved using this technique, and the results are shown in Figure 4.3. We find that the antenna pattern is broader in the H-plane than in the E-plane, because the antenna feed is narrow in the H-plane and wide in the E-plane.

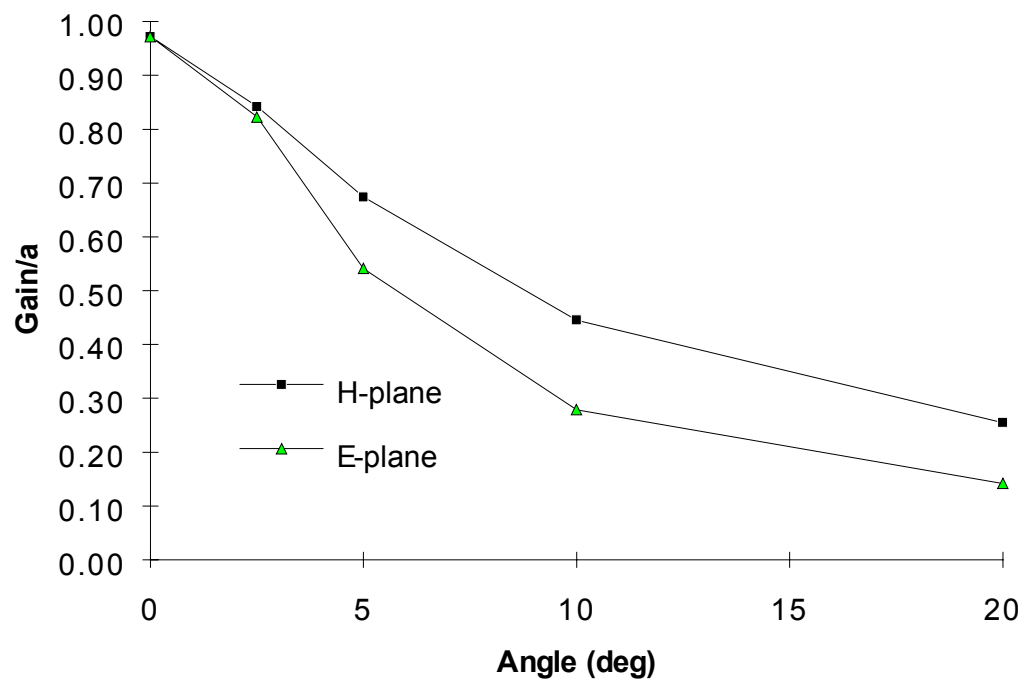

Figure 4.3. Gain ( $\infty$-norm) in the $\mathrm{H}$ - and E-planes as a function of angle. For this plot, $Z_{c}=400 \Omega$, and $T_{d}=0.1$ (e.g., $a=0.3 \mathrm{~m}$ and $t_{d}=0.1 \mathrm{~ns}$ ).

\section{SAMPLE MEASUREMENTS}

Finally, measurements of a reflector IRA, a lens IRA (Figure 1.1), and a TEM horn were made on a tabletop scale model ${ }^{17}$, where half the antenna was built on a ground plane. The lens IRA without the lens is just a simple TEM horn, so measurements were made both with and without the lens for comparison. The reflector was $58 \mathrm{~cm}$ in diameter with an $F / D$ of 0.48 , The reflector IRA had a single feed arm in the "facing plates" configuration with an input impedance of $200 \Omega$, which would correspond to $400 \Omega$ for a full model. The TEM horn/lens IRA was $61 \mathrm{~cm}$ in diameter at its aperture, and the length of the TEM horn/lens IRA feed section was $72 \mathrm{~cm}$. The feed impedance of the TEM horn/lens IRA was $94.25 \Omega$, which would correspond to $188.5 \Omega$ for a full antenna. The antennas was driven by a $40 \mathrm{~V}$ step function with a nominal $10-90 \%$ risetime of 100 ps. A " limited angle of incidence, limited time" sensor ${ }^{16}$ was used to detect the signal in replicative 
mode. The sensor was placed on boresight at a distance of $6.1 \mathrm{~m}$ from the aperture of the reflector IRA and $5.2 \mathrm{~m}$ from the aperture of the TEM horn/lens IRA.

A sample measured waveform for the reflector IRA is shown in Figure 5.1, along with theoretical predictions. One sees in this data a prepulse, an impulse, and an undershoot immediately following the impulse. The theory describing the undershoot is still being developed, but the theory of the prepulse and impulse already exist. When taking into account the feed blockage, there was a difference of five percent between the prediction and measurement of the waveform peak.

Sample measured waveforms for the lens IRA and TEM horn are shown in Figure 5.2, along with theoretical predictions for the lens IRA. Note that the lens provides about a factor of two improvement in the radiated field. Note also that the predications and measurements of the peak field for the lens IRA agree to within six percent.
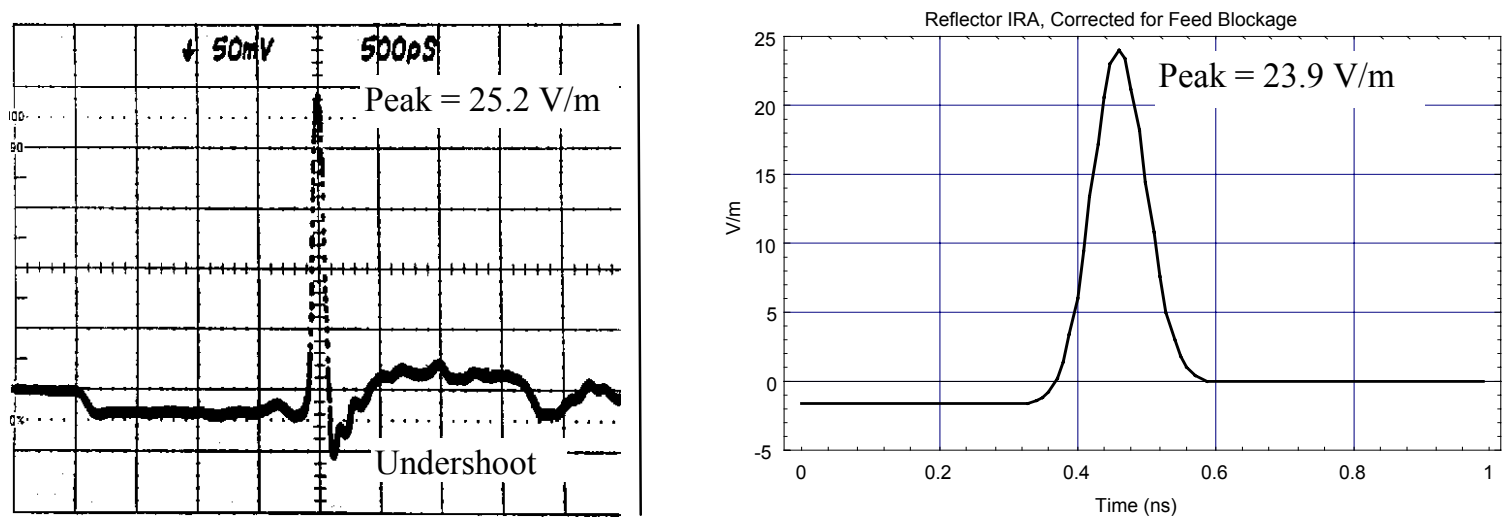

Figure 5.1 Experimental results (left) and predictions (right) for the reflector IRA. Note that the scales on the scope are $500 \mathrm{ps} /$ division horizontal and $50 \mathrm{mV} /$ division vertical. Since the effective height of the sensor is $0.95 \mathrm{~cm}$, this corresponds to a vertical scale of $5.26 \mathrm{~V} / \mathrm{m} /$ division.

TEM Horn

Peak $=27.3 \mathrm{~V} / \mathrm{m}$

Lens IRA

Peak $=59.9 \mathrm{~V} / \mathrm{m}$
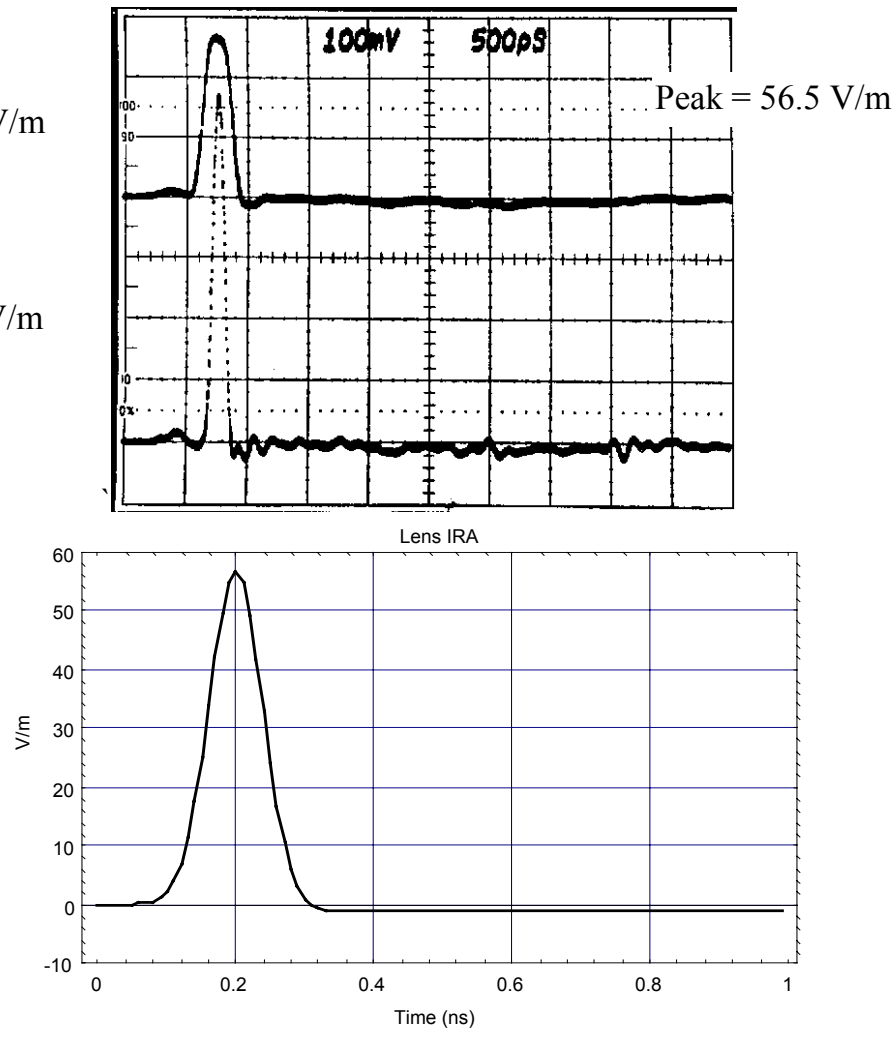

Figure 5.2 Experimental results (left) for TEM horn and lens IRA. Theoretical results for the lens IRA are on the right. Note that the scales for the measurements are $500 \mathrm{ps} /$ division horizontal and 100 $\mathrm{mV} /$ division vertical. Since the effective height of the sensor is $0.95 \mathrm{~cm}$, this corresponds to $10.5 \mathrm{~V} / \mathrm{m} /$ division vertical. 


\section{CONCLUSION}

We have considered here a number of extensions to the theory of IRAs. First, we developed a definition of gain that is as meaningful in reception mode as it is in transmission mode. This led to a radar equation in the time domain. Furthermore, we developed an approach for optimizing the feed impedance of reflector IRAs. We have also found a simple way of calculating the antenna pattern of reflector IRAs. Finally, we provided measurements which confirmed our predictions. 


\section{REFERENCES}

1. C. E. Baum and E. G. Farr, “Impulse Radiating Antennas,” pp. 139-147 in H. L. Bertoni et al (eds.), Ultra Wideband/Short-Pulse Electromagnetics, Plenum Press, New York, 1993.

2. E. G. Farr and J. S. Hofstra, An Incident Field Sensor for EMP Measurements, Sensor and Simulation Note 319, November 61989.

3. C. E. Baum, Radiation of Impulse-Like Transient Fields, Sensor and Simulation Note 321, November 1989. Also pp. 40-61 in P. Mayes (ed.), Proceedings of the 1993 Allerton Antenna Applications Symposium, RL-TR-94-20, February 1994.

4. C. E. Baum, Configurations of TEM Feed for an IRA, Sensor and Simulation Note 327, April 1991.

5. C. E. Baum, Aperture Efficiencies for IRAs, Sensor and Simulation Note 328, June 1991.

6. E. G. Farr, Analysis of the Impulse Radiating Antenna, Sensor and Simulation Note 329, July 1991.

7. C. E. Baum, General Properties of Antennas, Sensor and Simulation Note 330, July 1991.

8. E. G. Farr and C. E. Baum, Prepulse Associated with the TEM Feed of an Impulse Radiating Antenna, Sensor and Simulation Note 337, March 1992.

9. E. G. Farr and C. E. Baum, A Simple Model of Small-Angle TEM Horns, Sensor and Simulation Note 340, May 1992.

10. D. V. Giri, S. Y. Chu, On the Low-Frequency Electric Dipole Moment of Impulse Radiating Antennas (IRA's), Sensor and Simulation Note 346, October 1992.

11. E. G. Farr and C. E. Baum, Extending the Definitions of Antenna Gain and Radiation Pattern Into the Time Domain, Sensor and Simulation Note 350, November 1992.

12. C. E. Baum, Circular Aperture Antennas in Time Domain, Sensor and Simulation Note 351, November 1992.

13. E. G. Farr, Optimizing the Feed Impedance of Impulse Radiating Antennas, Part I: Reflector IRAs, Sensor and Simulation Note 354, June 1993. Also pp. 62-90 in P. Mayes (ed.), Proceedings of the 1993 Allerton Antenna Applications Symposium, RL-TR-94-20, February 1994.

14. E. G. Farr and C. E. Baum, Radiation from Self-Reciprocal Apertures, Sensor and Simulation Note 357, December 1993. Also to appear as Chapter 6 in C. E. Baum and H. L. Kritikos (eds.), Electromagnetic Symmetry, Taylor and Francis, (in publication) 1994.

15. E. G. Farr and C. E. Baum, The Radiation Pattern of Reflector Impulse Radiating Antennas: Early-Time Response, Sensor and Simulation Note 358, June 1993.

16. C. E. Baum, Limited-Angle-of-Incidence and Limited-Time Electric Sensors, Sensor and Simulation Note 359, June 1993.

17. E. G. Farr and C. J. Buchenauer, Experimental Validation of IRA Models, Sensor and Simulation Note 364, January 1994.

18. Antenna Standards Committee of the IEEE Antennas and Propagation Society, IEEE Standard Definitions of Terms for Antennas, published in IEEE Transactions on Antennas and Propagation, Vol. AP-31, No. 6, November 1983.

19. W. L. Stutzman and G. A. Thiele, Antenna Theory and Design, John Wiley \& Sons, 1981, p. 37.

20. O. E. Allen, et al, “ Time Domain Antenna Characterizations," IEEE Trans. Electromag. Compat. Vol. 35, August 1993, pp. 339-346.

21. R. W. Ziolkowski, " Properties of electromagnetic beams generated by ultra-wide bandwidth pulsedriven generators," IEEE Trans. Antennas and Propagat., Vol. 40, August 1992, pp. 888-905.

22. W. R. Smythe, Static and Dynamic Electricity, Third Ed., Hemisphere, 1989, p. 76 and p. 460.

23. C. E. Baum, Transient Scattering Length and Cross Section, Interaction Note 484, April 17, 1991.

24. C. E. Baum, Norms of Time-Domain Functions and Convolution Operators, Mathematics Note 86, December 9, 1985, and pp. 31-55 H. N. Kritikos and D. L. Jaggard, eds., Recent Advances in Electromagnetic Theory, Springer-Verlag, 1990, .

25. C. E. Baum and A. P. Stone, Transient Lens Synthesis, Taylor and Francis, New York, 1991.

26. C. E. Baum and A. P. Stone, "Transient Lenses for Transmission systems and Antennas," pp. 211-219 in H. L. Bertoni et al (eds.), Ultra Wideband/Short-Pulse Electromagnetics, Plenum Press, New York, 1993.

27. C. E. Baum, et al, A Uniform Lens for Launching a Spherical Wave into a Paraboloidal Reflector, Sensor and Simulation Note 360, July 1993. 